\title{
EVALUATION OF MYOCARDIAL CELL INJURIES AND OXIDATIVE STRESS MECHANISMS IN NEONATES AND INFANTS AFTER SURGICAL CORRECTION OF CONGENITAL HEART DISEASE
}

\author{
M.S. Oliveira ${ }^{1}$, E.Z. Martinez ${ }^{2}$, M.A. Rossi ${ }^{2}$, L.C. Perez ${ }^{3}$, S.G. Ramos ${ }^{3}$, E.M. Floriano ${ }^{2}$, S.C. Mazin ${ }^{2}$ \\ ${ }^{1}$ Pathology, University of Sao Paulo, Ribeirao Preto, ${ }^{2}$ University of Sao Paulo, Ribeirão Preto, Brazil, \\ ${ }^{3}$ Sheffield Teaching Hospitals NHS Foundation Trust, Sheffield, UK
}

Background and aims: Despite advances in pediatric cardiac surgery, perioperative myocardial injury can be the major determinant of postoperative dysfunction after cardiac surgery. The objective was to investigate the pathology-related differences in 29 infants with congenital heart disease that led to death.

Material and methods: The patients were divided into four groups: Group 1, 16 infants who underwent operations for congenital heart disease on cardiopulmonary bypass; Group 2, four infants who underwent off-cardiopulmonary bypass operations for congenital heart disease; Group 3, nine infants who died from congenital heart disease prior to surgical treatment; and Group 4 (control group), five infants with no congenital heart disease and who died from other causes. The myocardial injuries and oxidative stress mechanisms were assessed by histopathology and immunohistochemistry and were quantified by morphometrical analyses.

Results/conclusion: Contraction band necrosis and dystrophic calcification were found primarily in infants of Group 1. Coagulation necrosis and healing were prominent in Group 2, while infants without repair (Group 3) showed mainly colliquative myocytolysis. The control group showed no significant myocardial lesions. Lipid peroxidation was the principal mechanism of oxidative stress accounting for the myocardial lesions. The diversity of the lesions observed in these hearts seemed to indicate a large spectrum of cell damage due to inadequate myocardial perfusion, especially when these infants underwent surgery. Oxidative mechanisms could be a common mediator in the pathogenesis of myocardial injuries, resulting in changes in the permeability of the cell membrane, cell death, and intracellular calcium overload. 